\title{
The influence of life events on first and recurrent admissions in bipolar disorder
}

\author{
Sanne M Kemner ${ }^{1 *}$, Neeltje EM van Haren ${ }^{1}$, Florian Bootsman ${ }^{1}$, Marinus JC Eijkemans ${ }^{2}$, Ronald Vonk ${ }^{3}$, \\ Astrid C van der Schot ${ }^{4}$, Willem A Nolen ${ }^{5}$ and Manon HJ Hillegers ${ }^{1}$
}

\begin{abstract}
Background: Life events play an important role in the onset and course of bipolar disorder. We will test the influence of life events on first and recurrent admissions in bipolar disorder and their interaction to test the kindling hypothesis.

Methods: We collected information about life events and admissions across the life span in 51 bipolar patients. We constructed four models to explore the decay of life event effects on admissions. To test their interaction, we used the Andersen-Gill model.

Results: The relationship between life events and admissions was best described with a model in which the effects of life events gradually decayed by $25 \%$ per year. Both life event load and recurrent admissions significantly increased the risk of both first and subsequent admissions. No significant interaction between life event load and number of admissions was found.

Conclusions: Life events increase the risk of both first and recurrent admissions in bipolar disorder. We found no significant interaction between life events and admissions, but the effect of life events on admissions decreases after the first admission which is in line with the kindling hypothesis.
\end{abstract}

Keywords: Bipolar disorder; Mood disorder; Life events; Twins; Admissions

\section{Background}

The presence of psychopathology is often explained on the basis of stress-diathesis interactions (Monroe and Simons 1991). The diathesis-stress model serves to explore how non-biological or genetic traits (diatheses) interact with environmental influences (stressors) to trigger the onset of psychiatric disorders (Moffitt et al. 2005; Harris 2001). The environmental factor most frequently studied in this context is stress, often operationalized as life events. Numerous studies have demonstrated that life events play a role in the onset and course of both unipolar depression and bipolar disorder (Bender and Alloy 2011; Brown and Harris 1989; Hillegers et al. 2004; Hlastala et al. 2000; Malkoff-Schwartz et al. 1998).

Methodological limitations are a major issue when interpreting and comparing studies regarding the influence of

\footnotetext{
* Correspondence: s.m.kemner@umcutrecht.nl

${ }^{1}$ Brain Center Rudolf Magnus, Department of Psychiatry, University Medical Center Utrecht, A00.241, PO Box 85500, 3508 GA Utrecht, The Netherlands Full list of author information is available at the end of the article
}

life events on the onset and course of mood disorders (Johnson 2005). In many of these studies, data were obtained retrospectively, which complicates the reliable reporting of both life events and mood episodes due to recall bias. Moreover, regardless of the number of questions in an interview, people gradually forget life events (Paykel 1997; Brown and Harris 1982; Harris 2001). Furthermore, most studies so far used the number of episodes to define the course of illness whereas especially episodes longer ago are difficult to be remembered reliable, while it might be more reliable to report episodes which were associated with psychiatric admissions, as these are likely to reflect the most severe mood episodes and can often be confirmed with medical records.

The type of life event measures varies greatly between studies and poses another major obstacle in life event research. In particular, self-administered measures of stressful life events appear unreliable (Johnson 2005). Bender and Alloy (2011) confirmed that the gold standard of life stress measurements is the Life Events and

\section{它}


Difficulties Schedule (LEDS) (Brown and Harris 1978). The LEDS provides the opportunity to categorize, date and rate both positive and negative life events. Furthermore, in contrast to life event questionnaires, the LEDS interview includes both major and minor types of stress, making it more suitable for testing the kindling hypothesis where life events play a greater role in the onset of initial episodes than in subsequent later episodes, which can even occur more or less spontaneously (Post 1992). The kindling model was originally described as the electrical kindling in relation to epilepsy where after many repetitions of kindled seizures 'spontaneity' occurs, i.e. seizures develop in the absence of external stimulation (Pinel 1981; Wada et al. 1974). Interestingly, several studies have demonstrated that a history of episodes is a significant risk factor for future recurrences in mood disorders (Judd et al. 2008; Keller et al. 1983; Perlis et al. 2006). In bipolar disorder, several studies report that after the first admission, $50 \%$ to $75 \%$ of patients have a recurrence within 4 to 5 years (Bromet et al. 2005; Leverich et al. 2001).

So far, research on the kindling hypothesis has mainly focused on unipolar depression and a majority of studies indeed found supportive evidence (Bender and Alloy 2011). However, studies in bipolar disorder are limited and findings are inconsistent. Bender and Alloy (2011) integrated the current literature and showed that about half the studies failed to find evidence for the kindling hypothesis in bipolar disorder. Crucially, LEDS interview-based studies all failed to find such evidence (Dienes et al. 2006; Hammen and Gitlin 1997; Hlastala et al. 2000; Swendsen et al. 1995). However, they did establish significant associations between the onset and course of bipolar disorder and life events.

In an ongoing naturalistic longitudinal twin study on bipolar disorder, we obtained detailed life event information throughout the life span by using the LEDS and were able to look for possible associations with first and recurrent admissions. Our aims are (1) to assess the influence of the effect of life events on first and recurrent admissions; (2) to assess the influence of prior admissions on the risk of subsequent admissions; and (3) to test the interaction between life event load and number of admissions (i.e. as indication for a kindling effect) in those twins with bipolar disorder.

\section{Methods}

\section{Sample}

We conducted a secondary analysis with three a priori questions within an ongoing study among twins (affected twin pairs, $n=51$; healthy control twin pairs, $n=35$ ) with bipolar disorder at the University Medical Center Utrecht (UMCU), The Netherlands. Of this cohort, all 51 twins with bipolar disorder (bipolar I disorder, $n=37$; bipolar II disorder, $n=14$ ) were included in the current study. The design of the study and the recruitment of the bipolar twin pairs have been described in detail elsewhere (Van der Schot et al. 2009; Vonk et al. 2007). All participants were enrolled between 2001 and 2006. There were no restrictions on duration or stage of illness for inclusion in the study, and all patients were treated naturalistically.

Demographic information is displayed in Table 1. All diagnoses were confirmed with the Structured Clinical Interview for DSM-IV (First et al. 1996) and the Structured Interview for DSM-IV Personality (Pfohl et al. 1997). Hospitalizations were confirmed through available medical records. Current mood state was assessed using the Young Mania Rating Scale (YMRS; (Young et al. 1978)) and the Inventory for Depressive Symptomatology (IDS; (Rush et al. 1996)). At the time of the study, all patients were euthymic with a YMRS score of 4 or less and an IDS score of 12 or less.

The study was approved by the medical ethics review board of the University Medical Center Utrecht, and all participants gave written informed consent after full explanation of the study aims and procedures.

\section{Life event measures}

All subjects included in the current study were interviewed with the investigator-based Bedford College LEDS (Brown and Harris 1978, 1989). The LEDS is a semistructured interview for assessing life events and longterm difficulties in adults. It collects detailed information about the event itself, the timing of its occurrence (date) and relevant contextual information for each event. Each event is categorized into one of ten domains, consisting of education, work, reproduction, housing, money/possessions, crime/legal, health, marital/partner, other relationships and miscellaneous/death. Based on the contextual information, the threat for each event is rated via standardized rating procedures. The threat score represents the severity of the event, ranging from mild (1) to severe (4), hereby differentiating between mild life events and more stressful life events. The contextual threat is conceptualized as: 'What most people would be expected to feel about an event in a particular set of circumstances and biography, taking no account of what the respondent says either about his or her reaction or about any psychiatric or physical symptoms that followed it' (Brown and Harris 1989). Several studies have supported the reliability (e.g. interrater) and validity (e.g. multiple informant) of the LEDS in adults exhibiting a variety of psychiatric symptoms (Brown and Harris 1978, 1989; Ormel et al. 2001).

Only events occurring from the age of 5 years were included. All severe events were defined by the extent they were related to the bipolar disorder and to what extent they were dependent on the respondents' own behaviour. 
Table 1 Demographics

\begin{tabular}{|c|c|c|c|}
\hline & Total & Bipolar disorder type I & Bipolar disorder type II \\
\hline$n$ & 51 & 37 & 14 \\
\hline Female/male $(n)$ & $33 / 18$ & $25 / 12$ & $8 / 6$ \\
\hline Age at LEDS interview, $M(S D)$ & $40.49(9.52)$ & $39.51(8.71)$ & $43.07(11.33)$ \\
\hline Age at onset of the first bipolar episode, $M$ (SD) & $28.20(9.16)$ & $26.19(6.94)$ & $33.50(12.16)$ \\
\hline Age at onset of the first symptoms (all), $M$ (SD) & $26.08(8.85)$ & $25.27(6.82)$ & $28.21(12.86)$ \\
\hline Age at onset of treatment, $M(\mathrm{SD})$ & $27.72(9.03)$ & $26.41(7.71)$ & $31.46(11.59)$ \\
\hline Comorbid disorder (1, 2 or 3), $n(\%)$ & $11(22 \%)$ & $8(22 \%)$ & $3(21 \%)$ \\
\hline Psychotic symptoms lifetime, $n(\%)$ & $26(51 \%)$ & $24(65 \%)$ & $2(14 \%)$ \\
\hline \multicolumn{4}{|l|}{ Hospitalized group } \\
\hline Hospitalized patients, $n(\%)$ & $35(69 \%)$ & $31(84 \%)$ & $4(29 \%)$ \\
\hline Number of admissions, $M$ (SD) & $3.06(2.45)$ & $2.93(2.11)$ & $4.00(4.69)$ \\
\hline Age at first admission, $M(\mathrm{SD})$ & $27.91(7.86)$ & $26.61(6.4)$ & $38(11.83)$ \\
\hline \multicolumn{4}{|l|}{ Type of episode at first admission ( $n$ ) } \\
\hline Mania & 15 & 15 & 0 \\
\hline Depression & 12 & 9 & 3 \\
\hline Psychosis & 6 & 6 & 0 \\
\hline Others & 2 & 1 & 1 \\
\hline
\end{tabular}

To determine relatedness to the disorder, each severe event was rated on a three-point scale: 1) not related to psychopathology; 2) possibly related to psychopathology; or 3) clearly related to psychopathology. Only events with score 1 were included for further analyses. To determine if life events occurred independent of will or influence of the respondents' own behaviour, each severe event was rated on a seven-point scale: 1) completely independent; 2) nearly independent; 3) possible influence, however, very unlikely; 4) physical illness; 5) cooperation or agreement with external situation; 6) likely neglect or carelessness; and 7) intentional choice. Events rating 1 to 5 were included for further analyses. Each life event was dated per year. Age was then calculated for each event.

All interviewers and raters were trained by $\mathrm{MH}$, who was trained by G.W. Brown and T.O. Harris, who developed the LEDS. The interviews were conducted at the participant's home or at the UMCU. Events were rated by two independent raters who had not been involved in the interviews. A panel consisting of the four raters (including SK and $\mathrm{MH}$ ) reached consensus on the events that raised rating problems.

\section{Statistical analysis}

Life event load

Life event load represents the sum of the threat scores of the life events occurring in each year.

We calculated three different life event load measures: (1) cumulative load (CL), i.e. the life event load at a particular point in time (year $Y$ ) calculated as the sum of the life event load in year $Y$ and all preceding years; (2) cumulative load excluding events possibly or clearly related to the bipolar disorder (CL-NoBP); and (3) cumulative load including only independent events, thus excluding events possibly or clearly dependent on the respondents' own behaviour (CL-I).

Next, the life event load before the first or since the last admission was calculated. After each admission, life event load was reset to zero and was calculated as described above. The cumulative life event load in the year preceding the admission was used for analysis.

\section{Decay model}

Previous studies showed a decay effect, implying that the presumed effect of life events diminishes over time, e.g. the death of a close relative that occurred 3 or 4 years before admission has less impact compared to the same event 1 year before admission (Hillegers et al. 2004). We will investigate which decay model statistically fits the data best. To explore the degree to which the effect of life events diminishes over time, a time-specific life event load variable was calculated for every year and subjected to an exponential decay function. We tested four models; in model I, we tested the purely cumulative effect, and in models II to IV, the decay function implied a $25 \%, 50 \%$ and $75 \%$ loss of effect per year, respectively. The decay function yielding the best model fit $(-2 \times \log -$ likelihood) will be used for all further analysis.

\section{Andersen-Gill model}

The Andersen-Gill model (A-G model), an extension of the standard Cox proportional hazard model for recurrent 
events, accommodates censored data and time-dependent covariates (Fleming and Harrington 1991; Therneau and Grambsch 2000).

Data for the A-G model are structured such that for each individual, intervals at risk are defined by variables describing the start and end times of each year of age. An event variable is coded as ' 1 ' for admission and ' 0 ' for no admission.

The A-G approach follows the usual assumption of the Cox model that the hazard or risk ratio is proportional over time and more specifically that the risk of being admitted is unaffected by earlier admissions. Time-dependent covariates, such as the cumulative load of life events or the number of previous admissions, may be used to relax the latter assumption. The hazard ratio represents the proportionate change in the 'admission' rate due to a unit change in the respective covariate, in this case the cumulative life event load.

\section{Andersen-Gill model: interaction effect}

The presence of an interaction effect will be tested by integrating an interaction function in the A-G model, testing the effect of the interaction between the number of admissions and the cumulative load between the admissions in the best-fitted decay model, also known as a kindling effect (Post 1992).

\section{Results}

The general characteristics of our sample are shown in Table 1. At least one admission had occurred for 35 of the 51 bipolar patients, with a maximum of 11 admissions in two patients. Figure 1 and Table 2 display the number and polarity for all admissions.

\section{Influence of life event effect on first and recurrent admissions}

The relationship between life event load and admission (irrespective of the number of admissions) is depicted in Table 3. The exponentiated linear coefficients from the A-G model are interpreted as risk ratios relating the magnitude of a covariate (or multiple covariates) to admission. Positive coefficients indicate increased hazard for admission (' 1 ' vs ' 0 '). Figure 2 illustrates the cumulative life event load over time and the cumulative life event load between the admissions.

Independent of the model employed (cumulative, 25\%, $50 \%$ or $75 \%$ decay), the life event load was significantly associated with an increased risk of hospitalization per unit life event load. Adjustment for age and gender did not change the life event risk ratios. According to the log-likelihood, indicating the quality of fit, the decay model in which the life event load accumulates and at the same time decreases with a function of $25 \%$ with every subsequent year (model II) was most in agreement with the observed data. Therefore, all further analyses will be done under model II.

Table 4 displays the results of the A-G model with the three different types of load between the admissions: $\mathrm{CL}$, CL-NoBP and CL-I.

All coefficients for both life event load and number of admissions are positive and significant. Positive effect of all types of life event load indicates that the risk of getting admitted grows with an increasing life event load. This effect is independent of the type of life event load.

The A-G model with lifetime cumulative load and number of admissions shows a positive and significant risk ratio for both the lifetime load (coef $=.0985, \mathrm{SE}=.0166$, $p<.001$ ) and number of admissions (coef $=.4597, \mathrm{SE}=.0892$, $p<.001$ ), indicating that in addition to the cumulative load between the admissions, the lifetime cumulative load also contributes to the risk of getting admitted.

\section{Effect of number of previous admissions on admissions}

The positive and significant coefficient of the number of admissions on the risk of getting admitted implies an increase in the chance of getting admitted with each subsequent admission.

Interaction between life event load and number of admissions The interaction effect between the cumulative load between admissions in model II (with 25\% decay) and

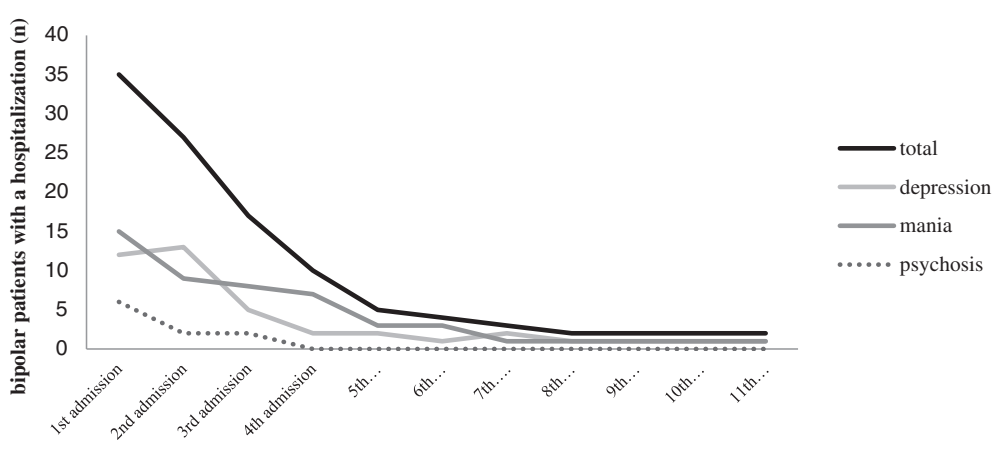

Figure 1 Number and polarity of admissions. 
Table 2 Number of admissions

\begin{tabular}{lc}
\hline Total number of admissions & $\boldsymbol{N}$ (total $\mathbf{= 5 1}$ ) \\
\hline 0 & 16 \\
1 & 8 \\
2 & 10 \\
3 & 7 \\
4 & 5 \\
5 & 1 \\
6 & 1 \\
7 & 1 \\
11 & 2 \\
\hline
\end{tabular}

number of admissions did not reach significance, indicating that the effect of cumulative load on the risk of admission does not change with subsequent admissions. However, the influence of life events on first admissions is higher compared with the influence of life events after on readmissions, suggesting a shift in the effect of life events between the first and subsequent admissions.

Results did not change when excluding the concordant co-twins from the sample. Also, neither age, age of onset of the first bipolar episode, age of first admission nor gender affected any of the above findings.

\section{Discussion}

Our main finding is that an increased life event load, taking into account the number and threat of life events, impacts both first and recurrent admissions in bipolar patients. This has also been found in previous studies (Bender and Alloy 2011; Hunt et al. 1992; Kessing et al. 1998, 2004), but it was hypothesized that this might be due to life events occurring as a consequence of the disease (Kessing et al. 2004). We now extended these previous findings by showing that the effect of life events on admissions did not change when events related to the disorder were excluded from the analyses. This suggests that the effect of life events is independent of life events occurring in relation to the disorder. We consider this robust influence of life events on first and recurrent admissions an important finding, as exposure and responses to life events are potentially modifiable. A better

Table 3 Relative risk of admission using four models of event effect decay

\begin{tabular}{lcccc}
\hline Model & Coefficient & Exp coefficient $^{\text {a }}$ & $\begin{array}{c}\text { Log-likelihood } \\
\text { of fitted model }\end{array}$ & $\boldsymbol{p}$ \\
\hline Cumulative & .024 & 1.024 & -308.5503 & $<.001$ \\
$25 \%$ decay & .134 & 1.143 & $-293.5533^{b}$ & $<.001$ \\
$50 \%$ decay & .240 & 1.272 & -298.4862 & $<.001$ \\
$75 \%$ decay & .452 & 1.572 & -306.3194 & $<.001$ \\
\hline
\end{tabular}

${ }^{\mathrm{a}}$ Exponentiated linear coefficients. ${ }^{\mathrm{b}}$ Lowest absolute log-likelihood of fitted model. understanding of how they impact the risk of being admitted may yield specific strategies for prevention and early intervention.

Our next finding was that the effect of the number of prior admissions on the risk of getting admitted was positive and significant, demonstrating that the risk increases with each admission. Several studies reported that after the first admission for bipolar disorder, $50 \%$ to $75 \%$ of patients relapse within 4 to 5 years (Bromet et al. 2005; Leverich et al. 2001). Our findings indicate that the risk of readmission increases as a function of the number of previous admissions. Given our finding that the risk of getting admitted is independent of events that are related to the disorder, such as admissions, the association between the number of previous admissions and increased risk of readmission might be interpreted as an indicator for illness severity. Moreover, this finding also suggests a possible kindling effect; previous admissions could trigger the next admission.

Finally, we found no significant interaction between life event load and the number of prior admissions on the risk to be readmitted, suggesting that the effect of life event load does not decrease as a function of subsequent admissions. However, we did find a stronger effect of life events on the first compared to subsequent admissions which does suggest a possible kindling effect. In this respect, it should however be realized that the kindling effect has mostly been found after the occurrence of five to seven episodes (Kendler et al. 2000; Kendler and Gardner 2001; Slavich et al. 2011) while we only looked at admissions and the average number of admissions lies between three and four in our sample. Previous studies using the LEDS in bipolar patients (Dienes et al. 2006; Swendsen et al. 1995; Hammen and Gitlin 1997) looking at episodes rather than admissions did not find evidence for either presence or absence of a kindling effect. This is in contrast with findings in unipolar depression, which might be explained by the more complex course of contrasting mood episodes (i.e. mania and depression) in bipolar disorder as compared with unipolar depression (only depression). Bipolar episodes can be manic, hypomanic, depressive or mixed, and it is possible that the influence of life events differs across these various episodes.

The effect of life events on admissions was best described by model II in which the influence of life events steadily accumulates (as one gets older, more life events occur) but at the same time gradually decays with $25 \%$ per year as time goes by (an event that has occurred years ago will no longer have the same impact as when it just happened). This decay model is in accordance with previous findings from our group in a sample of offspring of parents with bipolar disorder (Hillegers et al. 2004). The decay model of $25 \%$ (Figure 3) best 


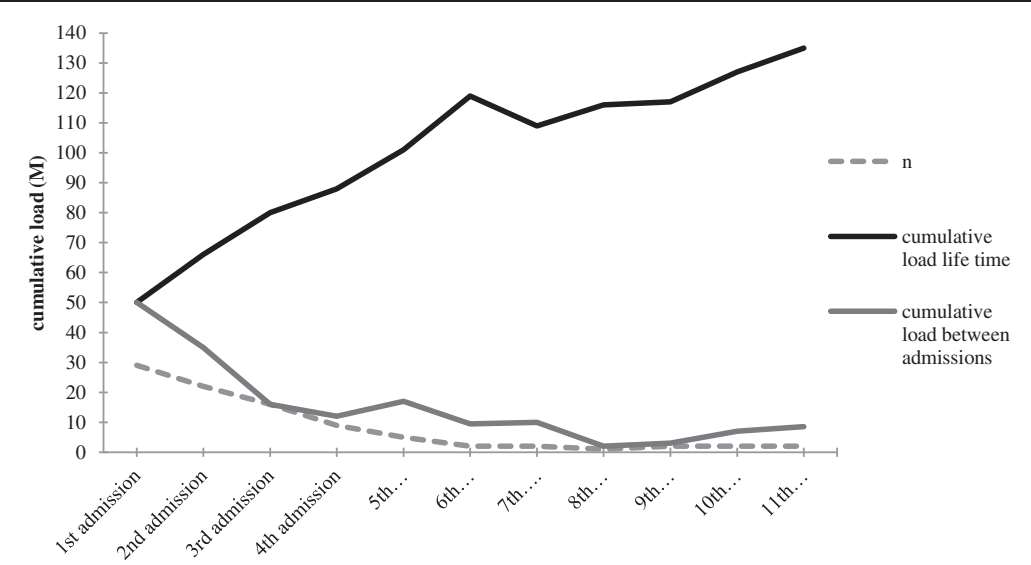

Figure 2 Course of cumulative load.

explained the influence of stressful life events on the onset of mood disorders when compared to the purely cumulative model or models with $50 \%$ or $75 \%$ decay per year. The underlying mechanisms that cause this decay are not known; a possible explanation lies in the interaction of life stress with coping strategies and temperament. Coping responses influence the association between stress and the onset of mood episodes. Temperamental traits influence individual coping styles and modify the impact of stressful life events on mood episode onset (Compas et al. 2004).
There are several limitations that need to be taken into account when interpreting our findings. Firstly, methodological limitations are a major issue when interpreting and comparing studies regarding the influence of life events on the onset and course of mood disorders (Johnson 2005). In many of these studies, information on life events was obtained retrospectively with queries or (semi-) structured interviews, which complicates the reliable reporting due to recall bias. Regardless of the number of queries in an interview, people gradually forget life events (Paykel 1997; Brown and Harris 1982; Harris 2001). The average

Table 4 Influence and interaction of types of cumulative load ( $25 \%$ decay), admissions and number of admissions

\begin{tabular}{|c|c|c|c|c|c|c|}
\hline & Coefficient & Exp coefficient $^{a}$ & SE (coef) & Robust $\mathrm{SE}^{\mathrm{b}}$ & $z$ & $p$ \\
\hline \multicolumn{7}{|c|}{ Type of cumulative load between admissions } \\
\hline $\mathrm{CL}$ & .086 & 1.09 & .019 & .021 & 4.17 & $<.001$ \\
\hline Number of admissions & .560 & 1.75 & .064 & .093 & 6.03 & $<.001$ \\
\hline$C L-1$ & .093 & 1.10 & .023 & .021 & 4.35 & $<.001$ \\
\hline Number of admissions & .577 & 1.78 & .066 & .099 & 5.81 & $<.001$ \\
\hline CL-NoBP & .085 & 1.09 & .027 & .024 & 3.59 & $<.001$ \\
\hline Number of admissions & .603 & 1.83 & .069 & .111 & 5.43 & $<.001$ \\
\hline \multicolumn{7}{|l|}{ Interaction effect } \\
\hline$C L$ & .071 & 1.07 & .027 & .026 & 2.79 & $<.001$ \\
\hline Number of admissions & .513 & 1.67 & .091 & .115 & 4.47 & $<.001$ \\
\hline $\mathrm{CL} \times$ number of admissions & .006 & 1.01 & .009 & .008 & 0.75 & .45 \\
\hline$C L-1$ & .081 & 1.08 & .030 & .023 & 3.49 & $<.001$ \\
\hline Number of admissions & .537 & 1.71 & .091 & .118 & 4.56 & $<.001$ \\
\hline CL-I $\times$ number of admissions & .006 & 1.01 & .009 & .010 & 0.58 & .57 \\
\hline CL-NoBP & .053 & 1.05 & .035 & .024 & 2.25 & $<.05$ \\
\hline Number of admissions & .510 & 1.67 & .093 & .125 & 4.07 & $<.001$ \\
\hline CL-NoBP $\times$ number of admissions & .018 & 1.02 & .012 & .015 & 1.23 & .22 \\
\hline
\end{tabular}

$\mathrm{CL}$, cumulative load including all events; CL-I, cumulative load including only independent events; CL-NoBP, cumulative load excluding events related to the disorder. ${ }^{\mathrm{a} E x p o n e n t i a t e d ~ c o e f f i c i e n t s, ~ r e p r e s e n t i n g ~ t h e ~ h a z a r d ~ r a t i o . ~}{ }^{\mathrm{b}}$ Robust standard error (SE), corrected for the dependency of multiple times to event within the same subject. 


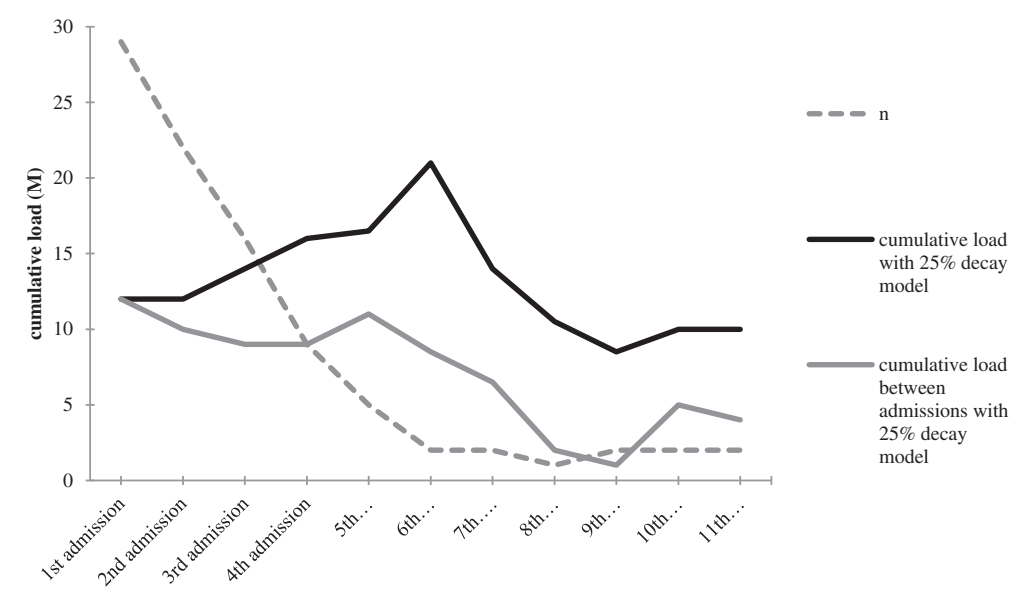

Figure 3 Course of cumulative load under the $25 \%$ decay model.

participant in our sample had to report life events over a time span of 35 years. One could question the reliability of the LEDS when it is used retrospectively to collect lifetime life event data. Most studies restrict the reporting of life events to a 12-month period. However, the LEDS is probably more reliable compared to (retrospective) checklist inventories (Hillegers et al. 2004; Ormel et al. 2001), as the LEDS minimizes recall bias; information is actively obtained in a very structured interview by detailed questions in ten domains. Furthermore, there is evidence that recall bias is more pronounced for minor events, suggesting that major life changes are under less influence of recall bias (Funch and Marshall 1984).

Secondly, more than one admission could occur per year. It is not clear to what extent this influenced our results, since admissions occurring within 3 to 6 months after the first admission are associated with more subclinical affective symptoms and therefore could be due to the same bipolar episode (Bromet et al. 2005). Unfortunately, the data on life events was dated per year and did not allow us to conduct the analysis in more detail.

We made no distinction between admissions due to mania, depression or psychosis. However, as can be seen in Figure 1, the polarity of the admissions is equally divided across the number of admissions for manic and depressive episodes.

Our sample is drawn from a longitudinal twin study. Having participants in the sample that share their genes and environment to a large extent might influence the study results. However, excluding the bipolar co-twins $(n=8)$ resulting in only one twin per pair in the analysis $(n=43)$ did not change our findings.

Finally, although most analyses yielded significant results, we have a small sample size consisting of patients with bipolar I as well as bipolar II disorders. So far, most studies limit their sample to bipolar type I (Bender and
Alloy 2011). The small sample size did not allow us to compare the two subtypes.

\section{Conclusions}

Life events, taking into account the number and threat of life events, appeared to have an impact on both first and recurrent admissions in bipolar patients, and this effect appeared not be dependent on events related to the illness. In addition, the number of prior admissions was positively related to the risk of getting readmitted. Finally, we did not find an interaction between life events and admissions on the risk for readmission, although the effect of life events was stronger on first admissions compared to readmissions, which suggests a possible kindling effect.

\section{Abbreviations}

A-G model: Andersen-Gill model; CL: cumulative load; CL-I: cumulative load including only events occurring independent of the respondents' own behaviour; CL-NoBP: cumulative load excluding all events occurring as a consequence of bipolar disorder; LEDS: Life Events and Difficulties Schedule.

\section{Competing interests}

$\mathrm{MH}$ has received grants from the Netherlands Organisation for Health Research and Development and the Brain and Behavior Research Foundation and has received speaker's fees from Astra Zeneca, Benecke, Shire and Lundbeck. WN has received grants from the Netherlands Organisation for Health Research and Development, the European Union, Astra Zeneca, GlaxoSmithKline and Wyeth, and has received honoraria/speaker's fees from Astra Zeneca and Lundbeck. The other authors report no competing interests.

\section{Authors' contributions}

SK carried out the data acquisition, data preparation and analyses, and drafted and edited the manuscript. NH participated in the design of the study and drafted the manuscript. FB carried out the data acquisition and data preparation and reviewed the manuscript. ME designed and carried out and assisted with statistical analysis. RV participated in the study design and data acquisition. AS participated in the study design and data acquisition. WA conceived the study and participated in its design and coordination. $\mathrm{MHJH}$ participated in the study design and drafted the manuscript. All authors read and approved the final manuscript. 


\section{Acknowledgements}

The authors wish to thank Annemiek van den Brand, Mirre Hubers, Guusje Havenaar, Nanja Hibbel and Afanaisa Lazo for assistance in conducting and rating the LEDS interviews.

\section{Financial support}

This study was supported by grant 22963 from the 7FP of the European Commission, grant 9120818 from the Netherlands Organisation for Scientific Research (NWO) and Stanley Medical Research Institute.

\section{Author details}

${ }^{1}$ Brain Center Rudolf Magnus, Department of Psychiatry, University Medical Center Utrecht, A00.241, PO Box 85500, 3508 GA Utrecht, The Netherlands. 2Julius Center for Health Sciences and Primary Care, University Medical Center Utrecht, Utrecht, The Netherlands. ${ }^{3}$ Reinier van Arkel Group, 's-Hertogenbosch, The Netherlands. ${ }^{4}$ Alzheimer Nederland, Amersfoort, The Netherlands. ${ }^{5}$ Department of Psychiatry, University Medical Center Groningen, University of Groningen, Groningen, The Netherlands.

\section{Received: 26 October 2014 Accepted: 26 January 2015}

\section{Published online: 25 February 2015}

\section{References}

Bender RE, Alloy LB. Life stress and kindling in bipolar disorder: review of the evidence and integration with emerging biopsychosocial theories. Clin Psychol Rev. 2011;31:383-98.

Bromet EJ, Finch SJ, Carlson GA, Fochtmann L, Mojtabai R, Craig TJ, et al. Time to remission and relapse after the first hospital admission in severe bipolar disorder. Soc Psychiatry Psychiatr Epidemiol. 2005;40:106-13.

Brown GH, Harris TO. Social origins of depression: a study of psychiatric disorder in woman. London: Tavistock Publications; 1978

Brown GH, Harris TO. Fall-off in the reporting of life events. Soc Psychiatr. $1982 ; 17: 23-8$

Brown GH, Harris TO. Life events and illness. New York: Guilford Press; 1989.

Compas BE, Connor-Smith J, Jaser SS. Temperament, stress reactivity, and coping: implications for depression in childhood and adolescence. J Clin Child Adolesc Psychol. 2004;33:21-31.

Dienes KA, Hammen C, Henry RM, Cohen AN, Daley SE. The stress sensitization hypothesis: understanding the course of bipolar disorder. J Affect Disord. 2006:95:43-9.

First MB, Spitzer RL, Gibbon M, Williams J. Structured clinical interview for DSM-IV axis I disorders- patient. 20th ed. New York: New York State Psychiatric Institute; 1996.

Fleming T, Harrington D. Counting processes and survival analysis. New York, NY, USA: Wiley; 1991

Funch DP, Marshall JR. Measuring life stress: factors affecting fall-off in the reporting of life events. J Health Soc Behav. 1984:25:453-64.

Hammen C, Gitlin M. Stress reactivity in bipolar patients and its relation to prior history of disorder. Am J Psychiatry. 1997;154:856-7.

Harris T. Recent developments in understanding the psychosocial aspects of depression. Br Med Bull. 2001;57:17-32.

Hillegers MH, Burger H, Wals M, Reichart CG, Verhulst FC, Nolen WA, et al. Impact of stressful life events, familial loading and their interaction on the onset of mood disorders: study in a high-risk cohort of adolescent offspring of parents with bipolar disorder. Br J Psychiatry. 2004;185:97-101.

Hlastala SA, Frank E, Kowalski J, Sherrill JT, Tu XM, Anderson B, et al. Stressful life events, bipolar disorder, and the 'kindling model'. J Abnorm Psychol. 2000;109:777-86.

Hunt N, Bruce-Jones W, Silverstone T. Life events and relapse in bipolar affective disorder. J Affect Disord. 1992;25:13-20.

Johnson SL. Life events in bipolar disorder: towards more specific models. Clin Psychol Rev. 2005;25:1008-27.

Judd LL, Schettler PJ, Akiskal HS, Coryell W, Leon AC, Maser JD, et al. Residual symptom recovery from major affective episodes in bipolar disorders and rapid episode relapse/recurrence. Arch Gen Psychiatry. 2008;65:386-94.

Keller MB, Lavori PW, Lewis CE, Klerman GL. Predictors of relapse in major depressive disorder. JAMA. 1983;250:3299-304.

Kendler KS, Gardner CO. Monozygotic twins discordant for major depression: a preliminary exploration of the role of environmental experiences in the aetiology and course of illness. Psychol Med. 2001;31:411-23.
Kendler KS, Thornton LM, Gardner CO. Stressful life events and previous episodes in the etiology of major depression in women: an evaluation of the 'kindling' hypothesis. Am J Psychiatry. 2000;157:1243-51.

Kessing LV, Andersen PK, Mortensen PB. Predictors of recurrence in affective disorder. A case register study. J Affect Disord. 1998;49:101-8.

Kessing LV, Agerbo E, Mortensen PB. Major stressful life events and other risk factors for first admission with mania. Bipolar Disord. 2004;6:122-9.

Leverich GS, Nolen WA, Rush AJ, McElroy SL, Keck PE, Denicoff KD, et al. The Stanley Foundation Bipolar Treatment Outcome Network. I. Longitudinal methodology. J Affect Disord. 2001;67:33-44.

Malkoff-Schwartz S, Frank E, Anderson B, Sherrill JT, Siegel L, Patterson D, et al. Stressful life events and social rhythm disruption in the onset of manic and depressive bipolar episodes: a preliminary investigation. Arch Gen Psychiatry. 1998;55:702-7.

Moffitt TE, Caspi A, Rutter M. Strategy for investigating interactions between measured genes and measured environments. Arch Gen Psychiatry. 2005:62:473-81.

Monroe SM, Simons AD. Diathesis-stress theories in the context of life stress research: implications for the depressive disorders. Psychol Bull. 1991;110:406-25.

Ormel J, Oldehinkel AJ, Brilman El. The interplay and etiological continuity of neuroticism, difficulties, and life events in the etiology of major and subsyndromal, first and recurrent depressive episodes in later life. Am J Psychiatry. 2001;158:885-91.

Paykel ES. The interview for recent life events. Psychol Med. 1997;27:301-10

Perlis RH, Ostacher MJ, Patel JK, Marangell LB, Zhang H, Wisniewski SR, et al. Predictors of recurrence in bipolar disorder: primary outcomes from the Systematic Treatment Enhancement Program for Bipolar Disorder (STEP-BD). Am J Psychiatry. 2006;163:217-24.

Pfohl B, Blum N, Zimmerman M. Structured interview for DSM-IV personality; SIDP-IV. lowa: American Psychiatric Press; 1997.

Pinel JP. Kindling-induced experimental epilepsy in rats: cortical stimulation. Exp Neurol. 1981;72:559-69.

Post RM. Transduction of psychosocial stress into the neurobiology of recurrent affective disorder. Am J Psychiatry. 1992;149:999-1010.

Rush AJ, Gullion CM, Basco MR, Jarrett RB, Trivedi MH. The Inventory of Depressive Symptomatology (IDS): psychometric properties. Psychol Med. 1996;26:477-86.

Slavich GM, Monroe SM, Gotlib $\Vdash H$. Early parental loss and depression history: associations with recent life stress in major depressive disorder. J Psychiatr Res. 2011;45:1146-52.

Swendsen J, Hammen C, Heller T, Gitlin M. Correlates of stress reactivity in patients with bipolar disorder. Am J Psychiatry. 1995;152:795-7.

Therneau TM, Grambsch PM. Modeling survival data: extending the Cox model. New York, NY, USA: Springer-Verlag; 2000.

van der Schot AC, Vonk R, Brans RG, van Haren NE, Koolschijn PC, Nuboer V, et al. Influence of genes and environment on brain volumes in twin pairs concordant and discordant for bipolar disorder. Arch Gen Psychiatry. 2009;66:142-51.

Vonk R, van der Schot AC, Kahn RS, Nolen WA, Drexhage HA. Is autoimmune thyroiditis part of the genetic vulnerability (or an endophenotype) for bipolar disorder? Biol Psychiatry. 2007;62:135-40.

Wada JA, Sato M, Corcoran ME. Persistent seizure susceptibility and recurrent spontaneous seizures in kindled cats. Epilepsia. 1974;15:465-78.

Young RC, Biggs JT, Ziegler VE, Meyer DA. A rating scale for mania: reliability, validity and sensitivity. Br J Psychiatry. 1978;133:429-35. 